\title{
Simulation Evaluation of Equivalent Vision Technologies for Aerospace Operations
}

\author{
Lynda J. Kramer*, Steven P. Williams, Susan J. Wilz, and Jarvis (Trey) J. Arthur III \\ NASA Langley Research Center, M/S 152, Hampton, VA 23681-2199
}

\begin{abstract}
A fixed-based simulation experiment was conducted in NASA Langley Research Center's Integration Flight Deck simulator to investigate enabling technologies for equivalent visual operations (EVO) in the emerging Next Generation Air Transportation System operating environment. EVO implies the capability to achieve or even improve on the safety of current-day Visual Flight Rules (VFR) operations, maintain the operational tempos of VFR, and perhaps even retain VFR procedures - all independent of the actual weather and visibility conditions. Twenty-four air transport-rated pilots evaluated the use of Synthetic/Enhanced Vision Systems (S/EVS) and eXternal Vision Systems (XVS) technologies as enabling technologies for future all-weather operations. The experimental objectives were to determine the feasibility of $\mathrm{XVS} / \mathrm{SVS} / \mathrm{EVS}$ to provide for all weather (visibility) landing capability without the need (or ability) for a visual approach segment and to determine the interaction of XVS/EVS and peripheral vision cues for terminal area and surface operations. Another key element of the testing investigated the pilot's awareness and reaction to non-normal events (i.e., failure conditions) that were unexpectedly introduced into the experiment. These non-normal runs served as critical determinants in the underlying safety of all-weather operations. Experimental data from this test are cast into performance-based approach and landing standards which might establish a basis for future all-weather landing operations. Glideslope tracking performance appears to have improved with the elimination of the approach visual segment. This improvement can most likely be attributed to the fact that the pilots didn't have to simultaneously perform glideslope corrections and find required visual landing references in order to continue a landing. Lateral tracking performance was excellent regardless of the display concept being evaluated or whether or not there were peripheral cues in the side window. Although workload ratings were significantly less when peripheral cues were present compared to when there were none, these differences appear to be operationally inconsequential. Larger display concepts tested in this experiment showed significant situation awareness (SA) improvements and workload reductions compared to smaller display concepts. With a fixed display size, a color display was more influential in SA and workload ratings than a collimated display.
\end{abstract}

Keywords: Synthetic Vision, Enhanced Vision System, External Visibility System, Head-Up Display, Global Positioning System, Aviation Safety, Supersonics, Next Generation Air Transportation System, Equivalent Visual Operations

\section{INTRODUCTION}

The U.S. air transportation system ${ }^{1}$ is undergoing a transformation to accommodate a projected threefold increase in air operations by 2025. One of the key capabilities envisioned to achieve this Next Generation Air Transportation System is the concept of equivalent visual operations (EVO). EVO implies the capability to achieve or even improve on the safety of current-day Visual Flight Rules (VFR) operations, maintain the operational tempos of VFR, and perhaps even retain VFR procedures - all independent of the actual weather and visibility conditions. The Integrated Intelligent Flight Deck (IIFD) project ${ }^{2}$, under NASA's Aviation Safety Program (AvSP), is investigating the use of synthetic and enhanced vision (S/EV) as enabling technologies to meet the challenges of EVO, creating a virtual visual flight environment for the flight crew, independent of the outside weather and visibility conditions.

Synthetic Vision (SV) is a computer-generated image of the external scene topography, generated from aircraft attitude, high-precision navigation, and data of the terrain, obstacles, cultural features, and other required flight information. Enhanced Vision (EV) is an electronic means to provide a display (typically on a head-up display, or HUD) of the external scene by use of an imaging sensor, such as a Forward-Looking InfraRed (FLIR) or millimeter wave radar.

*lynda.j.kramer@nasa.gov; phone 1757 864-8146; fax 1757 864-7793 
Prior research ${ }^{3,4}$ has shown that significant improvements in situation awareness (SA) without concomitant increases in workload and display clutter could be provided by the integration and/or fusion of SV and EV technologies for the pilotflying (on a HUD) and the pilot-not-flying (on a auxiliary display) during reduced visibility approach operations. The fused imagery showed unadulterated SV imagery on the initial approach. At $600 \mathrm{ft}$ height above touchdown (HAT), the SV image begins transitioning to an EV image by slowly fusing the SV and EV concepts. By $500 \mathrm{ft} \mathrm{HAT}$, the fusion process was completed and the resulting image was pure EV. The Pilot Flying (PF) followed existing FAA operating rules (Federal Aviation Regulation part 91.175) for using EV on a HUD, which require a transition from EV imagery to natural vision by $100 \mathrm{ft} \mathrm{HAT} \mathrm{to} \mathrm{continue} \mathrm{the} \mathrm{landing.} \mathrm{In} \mathrm{that} \mathrm{experiment,} \mathrm{crews} \mathrm{effectively} \mathrm{used} \mathrm{existing} \mathrm{EV} \mathrm{procedures}$ in their decision making, but found the required visual transition from an excellent EV image to natural vision by $100 \mathrm{ft}$ HAT to be awkward. Pilots commented that the EV imagery on the HUD provided suitable visual references to complete the flare and landing.

In addition to EVO, NASA is also investigating the use of vision technologies to overcome visibility limitations found in conceptual low-boom supersonic aircraft designs (Fig. 1). As is evident in Figure 1, pilot forward visibility using forward-facing windows would be severely compromised as a result of the vehicle configuration.

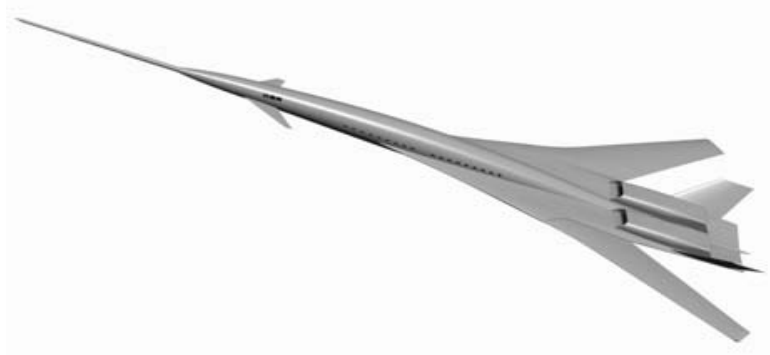

Figure 1. Conceptual low-boom supersonic aircraft configuration.

Under the Fundamental Aeronautics (FA) Program, Supersonics project, NASA is performing fundamental research, development, test and evaluation of flight deck and related technologies to support these low-boom, supersonic configurations by use of an eXternal Visibility System (XVS). XVS is a combination of sensor and display technologies which can provide an equivalent level of safety and performance to that provided by forward-facing windows in today's aircraft.

Without XVS, the economic viability of a low-boom supersonic aircraft is questionable, particularly since the lack of forward visibility by the pilot would severely restrict aircraft operations and airspace usage especially when the weather is clear and visibility conditions are unrestricted - i.e., when Visual Meteorological Conditions (VMC) prevail and visual approaches are possible. Without XVS, the crew of a low-boom supersonic aircraft cannot "see-and-avoid."

Significant research ${ }^{5,6}$ was conducted under NASA's High Speed Research (HSR) program during the 1990s on the design and development issues associated with an XVS for a conceptual high-speed civil transport aircraft. What emerged from this research (and still holds true today) is that the key challenge for an XVS design exists during VMC operations when it is assumed that the flight crew has natural visibility (even though they may be operating on an IFR flight plan).

Paradoxically, EVO technologies strive to create equivalent VFR operations for today's aircraft when flying in actual Instrument Flight Conditions (IMC) whereas XVS technologies try to enable an IMC cockpit (i.e., low-boom supersonic aircraft) to operate under VFR. The primary area of research overlap concerns the vision and visibility requirements to enable EVO or XVS. That is, what information and in what manner should this information be delivered to the flight crew to conduct EVO, whether EVO is created on the "windowed" flight deck when flying in actual IMC or whether it is created because of intentional restrictions to the flight crew's external visibility due to aircraft structure and design.

It has been hypothesized that the use of advanced vision technologies (such as S/EV and XVS) on conformal, head-up displays can provide precision approach, landing, and taxi guidance for "all weather" capability to all runways without requiring extensive approach lighting systems, ground-based precision guidance systems such as the instrument landing system (ILS), or other airport infrastructure. These technologies may provide "equivalent vision" capabilities which would obviate the need for airport lighting and other infrastructure to support the flight crews' need to visually acquire runways and taxiways using normal vision. 
An experiment which combined NASA flight deck research objectives from the IIFD Project/Crew-Vehicle Interface Element and the Supersonics Project was conducted to investigate the use of S/EV and XVS technologies as enabling technologies for future all-weather operations. The experimental objectives were:

- To determine the performance and safety of these advanced vision technologies for all weather (visibility) landing capability without the need (or capability) for a visual approach segment

- To evaluate if performance and safety for all weather landing capability is affected by whether the head-up display of information is provided using a collimated or un-collimated display

- To assess the interaction of head-up display information and the presence or absence of peripheral vision cues.

\section{METHODOLOGY}

\subsection{Subjects}

Twenty-four pilots, representing 6 airlines participated in the experiment. Each evaluation pilot (EP) had previous experience flying Head-Up Displays (HUDs). The EPs had an average of 1788 hours of HUD flying experience and an average of 20.4 years and 13.8 years of commercial and military flying experience, respectively.

\subsection{Simulation Facility}

The experiment was conducted in the Integration Flight Deck (IFD) simulation facility (Fig. 2) at NASA Langley Research Center (LaRC). The IFD emulates a Boeing B-757-200 aircraft and provides researchers with a full-mission simulator capability. The cab is populated with flight instrumentation and pilot controls, including the overhead subsystem panels, to replicate the B-757 aircraft. The collimated out-the-window (OTW) scene is produced by an Evans and Sutherland ESIG 4530 graphics system providing approximately 200 degrees horizontal by 40 degrees vertical fieldof-view at 26 pixels per degree. The forward windows were masked for this experiment but the side windows were unobstructed to allow testing of the effects of peripheral cues during approach, landing, taxi, and departure operations.

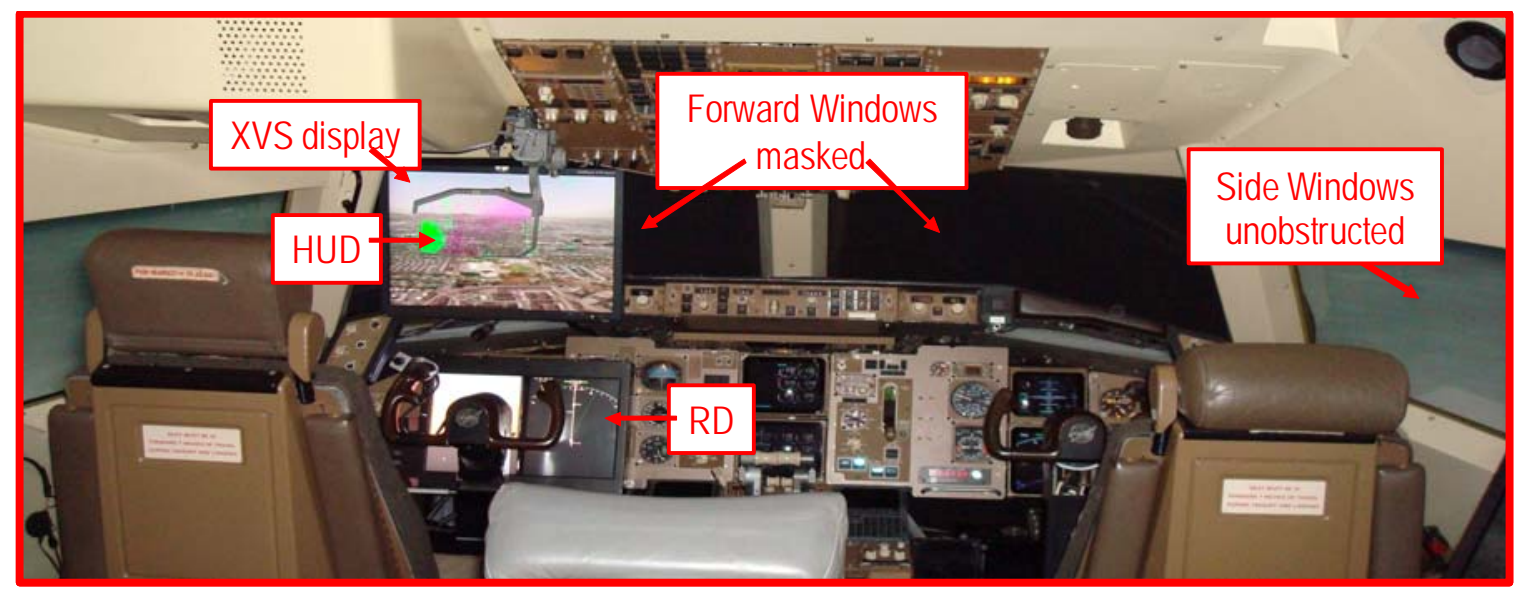

Figure 2. Integration Flight Deck simulation facility with HUD, XVS display and head-down Research Display (RD).

The EP occupied the left seat, as the Pilot Flying (PF). The left seat included an overhead HUD projection unit, a 20.1 inch diagonal Liquid Crystal Display (referred to as the XVS display) and a head-down research display (RD) (Fig. 2). The right seat was occupied by a principal investigator (PI) who acted as First Officer during data collection. The PI used his/her side windows to aid the EPs during surface operations by alerting them of upcoming right turns.

\subsubsection{Head-Up Display}

The HUD was collimated and subtended approximately $26^{\circ}$ horizontal by $21^{\circ}$ vertical field of view (FOV). Note that to maintain conformality with the outside world, the FOV for the HUD imagery was fixed and could not be varied by the EP. The HUD presentation was written strictly in monochromatic raster format from a video source (RS-343) input. The input consisted of a video mix of symbology and fused synthetic/enhanced vision (S/EV) imagery. The symbology included "haloing" to ensure that the symbology was highlighted against the scene imagery background. Overall HUD 
brightness and contrast controls were provided to the pilot. In addition, the EP was able to independently adjust the flight symbology brightness relative to the raster imagery. The pilot also had a declutter control, implemented as a fourbutton castle switch on the left hand horn of the PF yoke. Four "declutter" states were available to the EP: 1) Symbology toggle (on/off); 2) Imagery toggle (on/off); 3) All decluttered (no symbology or imagery); and 4) All symbology and imagery restored.

\subsection{2. eXternal Visibility System Display}

The XVS display subtended approximately $44^{\circ}$ horizontal by $34^{\circ}$ vertical FOV and was located approximately 19 inches from the pilot design eye point. The imagery on the XVS display was conformal with the OTW view just as the HUD imagery. However, this display differed from HUD as it was larger, used color, and was not collimated. The XVS display emulated a camera view mounted on the outside of the aircraft with flight symbology overlaid on the scene. Thus, any items (e.g., traffic, approach lighting system, terrain, runway markings, etc.) that would be visible to a real camera system would be visible in the color camera imagery. For experimental purposes, this photo-realistic camera imagery was unaffected by the outside weather to test for display size and peripheral cues effects. The same declutter control described in the HUD section above was utilized with the XVS display.

\subsubsection{Head-Down Research Display}

A head-down RD was installed over the normal instruments on the left hand side of the IFD cockpit (Fig 2). The RD covered the captain's displays normal for a Boeing 757-200. The RD used an 18.1-inch diagonal case containing two high brightness LCDs. The two separate LCD panels, each with XGA (1024x768) resolution, rendered a Size D (6.4 inch square viewable area) primary flight display (PDF) and navigation display (ND).

\subsection{Symbology}

The same symbology set was used for the XVS and HUD concepts (Fig. 3). The symbology included pathway guidance and a runway outline. The pathway symbology ${ }^{7}$ ended at $500 \mathrm{ft}$ HAT and was replaced by a runway outline and a glideslope reference line. A runway outline symbol $(8000 \mathrm{ft} \times 200 \mathrm{ft})$ was drawn using the threshold coordinates of the landing runway and the aircraft navigation solution to conformally position the symbol. A glideslope reference line was drawn at a descent angle of 3.1 degrees. Also, radar altitude was shown digitally underneath the flight path marker when below $500 \mathrm{ft}$ above ground level (AGL).

A pitch-roll guidance cue ("ball") used modified pursuit guidance ${ }^{8}$ along the desired path. Horizontal and vertical position of the ball reflects the track and flight path angles to fly to the center of the desired path. The path deviation indicators showed angular course deviation (i.e., glideslope and localizer-like) conditions by converting the linear path error data to angle errors and scaling in "dots." Glideslope and localizer raw data indicators which included a deviation scale and angular deviation indication were also provided (i.e., glideslope and localizer deviation).

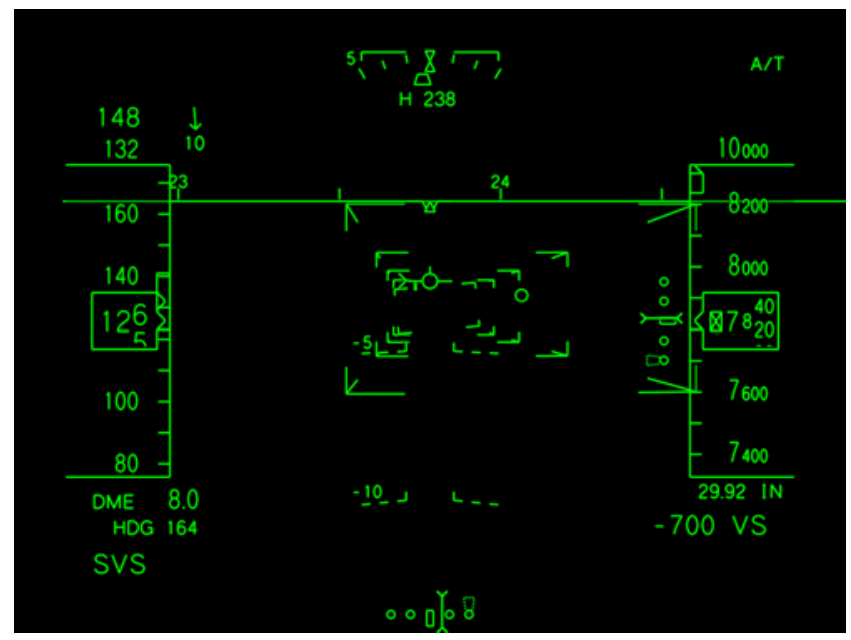

Figure 3. Head-up flight display symbology format.

\subsection{Display Concepts}

Three head-up flight display concepts were evaluated by the EPs while flying approaches to Runway 16R at the RenoTahoe International Airport (airport identifier RNO). The head-down PFD and ND formats were invariant. 
Three additional head-up display conditions were tested but not reported herein. These conditions were created by introducing a pitch bias which simulated an increased angle-of-attack. The nominal cases reported herein used a HUD pitch bias of 3 degrees which was tailored to the B-757 simulator and its nominal operating approach and landing conditions. The additional head-up display conditions used an 11 degree pitch bias to approximate that of a supersonic transport aircraft on approach.

\subsubsection{Head-Up Flight Display Concepts}

In Figure 4 (left picture), the HUD concept is shown for the low angle of attack (AOA) condition reported herein. The XVS display was turned off when the EP was evaluating a HUD concept. Note that the forward windows were masked for the HUD concept.

Two XVS display concepts (fig. 4, center and right pictures) were tested. The two XVS display concepts were created by simulating two different FOVs: 1) either the 26 × 21 degrees - identical to the HUD FOV; or, 2) the full FOV of the XVS display (44 x 34 degrees). The symbology used in the XVS concepts was identical to that used in the HUD concept.

Simulated color camera imagery was mixed with the symbology and shown conformally on the color, uncollimated XVS display for both FOVs. The HUD was stowed to preclude blocking or distortion of the pilot's forward view when using the XVS display. In Figure 4. both XVS display concepts are shown for the low AOA pitch attitude reported herein.
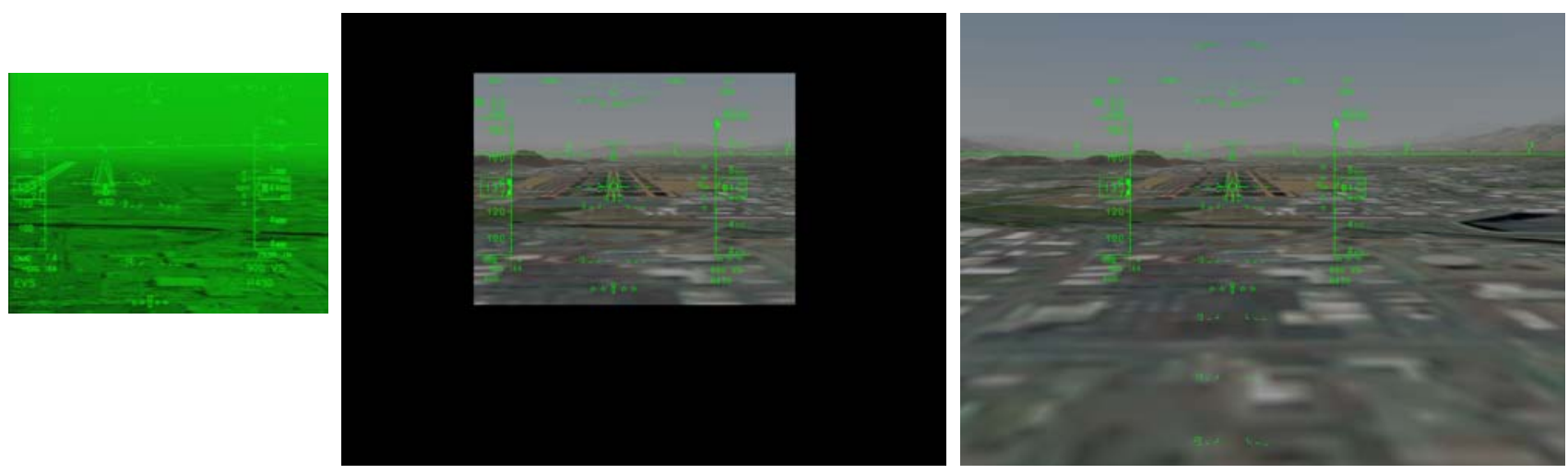

Figure 4. Head-up display formats - HUD (left), XVS with HUD FOV (center), and XVS with full FOV (right).

\subsubsection{Head-Down Flight Display Concepts}

The PFD and ND closely resembled typical transport aircraft equipage for glass cockpits. However, guidance information was purposely removed from the PFD so that the EPs would focus on the head-up flight display concepts. The ND showed the RNO Runway 16R approach path, but it did not include any Enhanced Ground Proximity Warning System nor Traffic Alert and Collision Avoidance System information.

\subsection{Approach Lighting System Configurations}

Three different approach lighting system (ALS) configurations were used for this experiment. The first ALS configuration (hereinafter referred to as the VFR ALS) was representative of lights found at a VFR runway and included Runway Edge Identification Lights (REIL), precision approach path indicator lights (PAPI), partial threshold lights, and medium intensity runway lights (MIRL). The second ALS configuration (hereinafter referred to as the MALSR ALS) was representative of a Category I/Type I operations runway and included MALSR (medium intensity approach lighting system; runway alignment indicator lights), PAPI, full threshold lights, and MIRL. The third ALS configuration (hereinafter referred to as the ALSF-2 ALS) was representative of a standard Category II/Type II operations runway and included ALSF-2 (high intensity approach lighting system with sequenced flashing lights), touchdown zone and centerline lighting, PAPI, full threshold lights, and high intensity runway lights (HIRL).

\subsection{Evaluation Task}

The approach task mimicked an existing visual arrival procedure reflecting an efficient and preferred routing for air traffic control and noise abatement. This approach requires visual meteorological conditions for the crew to see and 
avoid terrain, traffic, and obstacles while navigating with respect to ground references. The approach was a curved, descending path around terrain and obstacles and, thus, tests the ability of the display concepts to support this type of equivalent visual operation. The weather consisted of altitude-based cross winds (wind direction and intensity was dependent on altitude), light turbulence, and varying visibility levels ( 3 miles or $200 \mathrm{ft}$ runway visual range, RVR). The EP hand-flew the base and final legs of the visual arrival to RNO Runway 16R, using the HUD or XVS display concept with autothrottles engaged, holding 132 knots. The aircraft was configured for landing (landing gear down and flaps 30 degrees) prior to each run, and the aircraft was "cleared to land". The path converged into the instrument landing system approach course, nominally resulting in a stabilized approach no lower than 1,000 ft HAT. EPs were instructed that the approach runs would end with a full stop but that a go-around should be performed if they felt the approach or landing was not safe. The pilot was instructed to follow a pre-briefed taxi clearance requiring the aircraft to exit the runway on a high-speed turnoff onto Taxiway November, turn right on Taxiway Alpha, cross over Runway 7/25, and then turn left on Taxiway Lima where the run ended.

EPs also performed departures flying the RNO "Mustang 7" Departure Procedure. They maintained the runway heading of 168 degrees until waypoint RIJTU (about $5 \mathrm{nmi}$ from the departure runway) and then turned left direct toward the Mustang VORTAC, where the run ended. The weather consisted of altitude-based cross winds (wind direction and intensity was dependent on altitude), light turbulence, and varying visibility levels ( 3 miles or $200 \mathrm{ft} \mathrm{RVR}$ ). The EP hand-flew the departure with the HUD, XVS with HUD FOV or XVS with Full FOV display concept and was instructed to climb to $10,000 \mathrm{ft}$ mean sea level (MSL) and 250 knots.

There were up to 3 transport-sized aircraft in the runway environment, but they did not provide any conflicts for the ownship during approach, landing, taxi, or departure operations. There was no ATC involvement in the tasks.

\subsection{Pilot Procedures}

Since only pilot-flying evaluations were being conducted, automatic aural altitude call-outs (e.g., 1000, 500, 100 feet, etc.) were included in the simulation to "assist" in altitude awareness. Unlike current FAA regulations when landing with EV imagery on a HUD, for this experiment, the EP was not required to see using natural vision the required landing visual references (as per FAR §91.175) by $100 \mathrm{ft} \mathrm{HAT}$. The EP was instructed to continue to landing if the required landing visual references were seen in the imagery on the HUD or XVS and if the EP determined that a safe landing could be performed. Otherwise, a missed approach should be executed.

The EPs were instructed to fly the aircraft as if there were passengers aboard, fly the center of the approach path (within $\pm 1 / 2$ dot for desired performance and within \pm 1 dot for adequate performance), and land as close as possible to the centerline and aim point (1000 feet from the threshold). After landing, they were to capture the center line and then, taxi at a speed with which they were comfortable using the pre-briefed taxi clearance. They were also instructed to initiate a go-around if the landing was not safe or if there were any safety concerns during the approach. EPs were instructed to stop the aircraft if they felt unsafe during surface operations.

Prior to run commencement, the EP was briefed on the type of run to be completed, the display concept to be evaluated, the visibility level, and the wind magnitude and direction.

\subsection{Experiment Matrix}

Nominally, ten training runs and twenty-seven experimental runs were completed by each EP. Of the 27 experimental runs, 5 non-normal runs were included to investigate the pilot's awareness and reaction to unexpected events and conditions (e.g., failures). The non-normal data are critical determinants in the underlying safety of all-weather operations. Due to paper page-limit constraints, these data and also six of the 22 nominal runs (i.e., the so-called "high angle of attack" approaches) are not reported herein.

For approach and landing runs, the experiment matrix (Table 1) consisted of a full-factorial combination of display type (HUD, XVS with HUD FOV, or XVS with Full FOV) and peripheral cues in side windows. The peripheral cues were either absent, simulating Instrument Meteorological Conditions (IMC) of 200 RVR, or present, simulating Visual Meteorological Conditions (VMC) of 3 miles visibility. The MALSR ALS was used for these approach and landing runs.

Four additional runs were conducted to test for display type (HUD or XVS with Full FOV) and ALS (VFR, ALSF-2) effects. The IMC visibility level was used for these runs. These four runs are compared with 2 runs (HUD and XVS with Full FOV using MALSR ALS) from the Table 1 experimental matrix to test for ALS effects. 
For departure runs, the experiment matrix (Table 1) consisted of a full-factorial combination of display type and peripheral cues in the side windows for a total of 6 runs.

Table 1. Approach and departure run matrix. (evaluated using MALSR approach lighting system)

\begin{tabular}{|c|c|c|c|}
\hline & HUD & XVS with HUD FOV & XVS with Full FOV \\
\hline \hline IMC Approach and Landing & $\checkmark$ & $\checkmark$ & $\checkmark$ \\
\hline VMC Approach and Landing & $\checkmark$ & $\checkmark$ & $\checkmark$ \\
\hline IMC Takeoff & $\checkmark$ & $\checkmark$ & $\checkmark$ \\
\hline VMC Takeoff & $\checkmark$ & $\checkmark$ & $\checkmark$ \\
\hline
\end{tabular}

Note: VMC is visual meteorological conditions and IMC is instrument meteorological conditions.

A significant component of the test, in addition to the nominal runs, was the investigation of the ability of the EP to recognize and properly handle non-normal events. The number of non-normal scenarios was designed to avoid expectancy on the part of the flight crew. ${ }^{11}$ Five non-normal runs were flown by each EP. The non-normals were two flight director failures (annunciated and unannunciated), two flare cue failures (annunciated and unannunciated), and one rejected takeoff. The EPs were trained to recognize the annunciated flight director failure, annunciated flare cue failure, and rejected takeoff. For the failures on approach, they were instructed to continue the landing if they felt it was safe to do so. For the rejected takeoff, they were instructed to stop the aircraft as quickly as possible on the runway. For the annunciated failure runs, the affected symbol (flight director or flare cue) was removed from the display and a failure flag was presented on the display. For the unannunciated failure runs, the affected symbol was frozen on the display and no failure flag was presented on the display. The rejected takeoff run was caused by a simulated right engine fire annunciated aurally. A between-subjects design was employed for the non-normal approach and departure runs. As mentioned before, the results of the non-normal runs are not reported herein but will be reported elsewhere.

\subsection{Measures}

During each approach and landing run, path error, pilot control inputs, and touchdown performance (sink rate at touchdown, distance fore or aft of touchdown zone, and distance left or right of centerline) were measured for analysis. During taxi operations, centerline tracking and taxi speed were measured. For departure runs, centerline tracking, heading and climb rate maintenance, and altitude capture were measured.

After each run, pilots completed a run questionnaire consisting of the NASA Task Load Index (TLX) workload rating ${ }^{9}$, Situation Awareness Rating Technique (SART) ${ }^{9}$, and six Likert-type (5-point) questions specific to different constructs of making a stabilized and safe approach to landing, taxiing (when appropriate), or departure.

After data collection was completed, pilots were administered two paired comparison tests: the Situation Awareness Subjective Workload Dominance (SA-SWORD) ${ }^{10}$ and Subjective Workload Dominance (SWORD) ${ }^{9}$ techniques. The pilots also completed a post-test questionnaire to elicit comments on using the different display concepts, with and without peripheral cues, for conducting 1) approaches without a visual segment, 2) surface operations and 3) departures.

\subsection{Test Conduct}

The subjects were given a 1-hour briefing to explain the experiment purpose, HUD and XVS concepts, pilot procedures, and the evaluation tasks. After the briefing, a 1-hour training session in the IFD was conducted to familiarize the subjects with the aircraft handling qualities, display symbologies, pilot procedures, and controls. The annunciated flight director and flare cue failure runs and rejected landing runs were discussed and trained. The pilot's responsibility for maintaining safe operations at all times was stressed. Data collection lasted approximately 4.5 hours and was followed by debriefings which included the SWORD/SA-SWORD paired comparisons tests and a final questionnaire. The entire session including lunch and breaks lasted approximately 8 hours.

\section{RESULTS}

The objective and subjective results are presented for the low AOA display simulation conditions only. The comparison of high and low AOA effects will be reported elsewhere.

For the objective performance measures and post-run questions, analysis of variance (ANOVA) analyses were conducted for the factors of display type (HUD, XVS with HUD FOV, XVS with full FOV) and peripheral cues (absent, present). 
For the post-test paired comparisons, simple ANOVAs were conducted for display type. When necessary, StudentNewman-Keuls (SNK) post-hoc tests with $\alpha$ set at 0.05 were performed.

\subsection{Objective approach standards analysis}

The Joint Aviation Authorities (JAA) Joint Aviation Requirement (JAR) All Weather Operations (AWO) performancebased approach standard for go-around rate (AWO-202) in approaches with decision heights below $200 \mathrm{ft}$ and down to $100 \mathrm{ft}$ was applied in the objective data analysis ${ }^{12}$. Specifically, the standards specify that no more than $5 \%$ of the approaches will have localizer tracking greater than 1/3 dot or glideslope tracking greater than 1 dot between $300 \mathrm{ft}$ and $100 \mathrm{ft} \mathrm{HAT}$ for certification acceptance. These standards were not written specifically as quantitative performance standards for advanced vision systems (S/EV and XVS) operations, but are applied herein for comparative purposes.

The Continuous Method ${ }^{12}$ technique was employed to calculate the probability of success, $P(\alpha)$, of meeting the AWO exceedance criteria ( $1 / 3$ dot localizer, 1 dot glideslope) with required levels of confidence with the different display concepts flown. The probabilities of success for meeting the AWO localizer and glideslope criteria are shown, broken down by display configuration and visibility condition, in Table 2 .

Table 2. Probabilities of success in meeting the AWO localizer and glideslope criteria without a visual landing segment.

\begin{tabular}{|c|c|c|c|c|c|}
\hline Display & Weather & Localizer P( $\boldsymbol{\alpha})$ & Glideslope P( $\boldsymbol{\alpha})$ & Number of Runs & Number of Go-Arounds \\
\hline \hline HUD & VMC & 100.0 & 78.2 & 24 & 0 \\
\hline HUD & IMC & 100.0 & 67.4 & 24 & 0 \\
\hline XVS HUD FOV & VMC & 100.0 & 94.3 & 24 & 0 \\
\hline XVS HUD FOV & IMC & 100.0 & 80.6 & 24 & 1 \\
\hline XVS Full FOV & VMC & 100.0 & 96.0 & 24 & 0 \\
\hline XVS Full FOV & IMC & 100.0 & 92.6 & 24 & 0 \\
\hline
\end{tabular}

Note: Approaches flown using low angle of attack pitch attitude and MALSR approach lighting system.

The data in Table 2 shows that localizer tracking was maintained, irrespective of the display being flown or the absence or presence of peripheral cues in the side windows. However, glideslope tracking was better under the following conditions: 1) when flying with the larger FOV display concepts compared to the smaller (HUD) FOV display concepts, 2) with the color XVS HUD FOV display concepts compared to the monochrome HUD display concepts, and 3) when peripheral cues were present for the display concept. The only display concept successfully meeting the JAR AWO-202 localizer and glideslope criterion (greater than $95 \%$ of the time) was the XVS Full FOV display concept flown with peripheral cues present in the side windows. This color, uncollimated, display concept showed conformal imagery over a $34^{\circ}$ vertical FOV.

The Continuous Method was applied to data from a prior experiment ${ }^{13}$ for comparison. In this experiment, pilots evaluated the use of SV on a HUD during an instrument approach but they were required to transition to a natural vision segment, just as they would have to do today under existing operating regulations. (Note that the symbology used during the SV-HUD experiment was identical to that used in the current experiment, but the approach was flown at 138 knots instead of 132 knots.) The analysis revealed that $100 \%$ of the runs met the localizer criteria but only $60 \%$ of the runs met the glideslope criteria. The comparison of the two experiments shows that slight improvements in glideslope tracking may be realized with elimination of the visual segment (60\% vs. 67\%). The differences are not large, but the trends make intuitive sense. By allowing the pilot to concentrate on the task and display information, improved flight tracking performance results. The visual segment transition forces the pilots to simultaneously perform glideslope corrections and acquire the required landing visual references through normal vision.

\subsection{Objective landing standards analysis}

Existing JAR AWO ${ }^{12}$ performance-based landing standards (AWO 131) for longitudinal position and lateral position from centerline were applied in the objective landing data analysis. Specifically, the standards state that no longitudinal touchdown earlier than a point on the runway $200 \mathrm{ft}$ from the threshold or beyond $2700 \mathrm{ft}$ from the threshold and no lateral touchdown with the outboard landing gear more than $70 \mathrm{ft}$ from the runway centerline to a probability of $1 \times 10^{-6}$. These standards pertain to the general concept of low-visibility approach and landings, but were not written specifically for operations with advanced vision systems such as S/EV or XVS. 
This experiment used an aim point located $1000 \mathrm{ft}$ from runway threshold. For the simulated 757 aircraft, the outboard landing gear would be $70 \mathrm{ft}$ from the centerline when the fuselage (the recorded lateral landing position reported herein) is at $58 \mathrm{ft}$ lateral deviation from centerline, assuming no crab angle at touchdown.

In Figure 5, the touchdown data are shown, broken out by display concept but collapsed across peripheral cue influence. Included on this plot (in red, dashed rectangle) is the $+/-58 \mathrm{ft}$ lateral and $200 \mathrm{ft}$ to $2700 \mathrm{ft}$ longitudinal touchdown zone definition used in the JAR AWO landing criteria.

Statistical analysis against the AWO landing criteria meeting the $1 \times 10^{-6}$ probability requirements has not been completed, but visual inspection of the data shows that both XVS display concepts regardless of their FOV were within the JAR AWO touchdown zone definition.

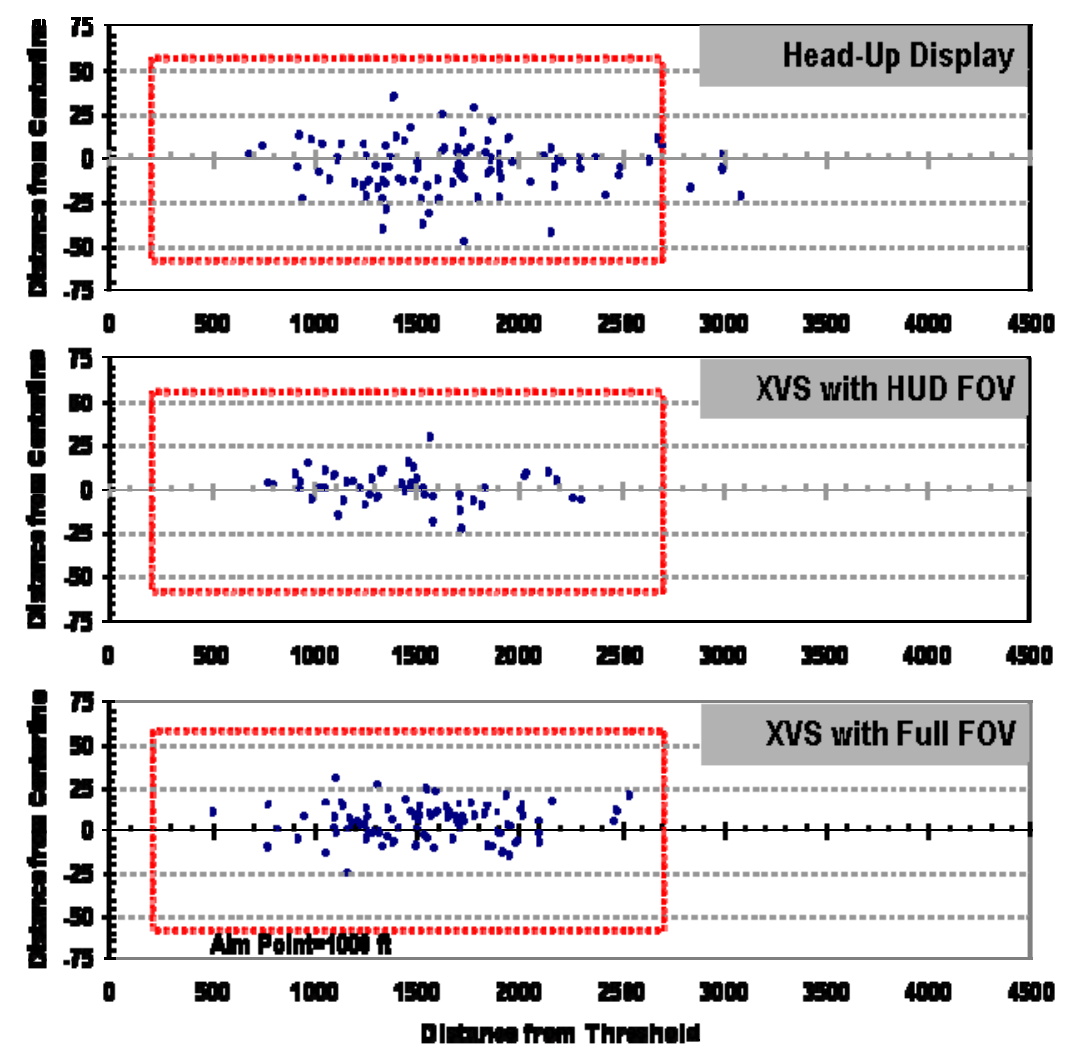

Figure. 5. Landing performance data for HUD (top), XVS with HUD FOV (middle), and XVS with large FOV (bottom).

\subsection{Mental Workload}

Mental workload was assessed after each experimental run, using the NASA TLX, and post-test, using the SWORD technique.

NASA TLX is a multi-dimensional rating procedure that derives an overall workload score based on a weighted average of ratings on six subscales (mental demand, physical demand, temporal demand, performance, effort, and frustration level). The NASA TLX subscales went from 0 (Low) to 100 (High) for the workload ratings. TLX ratings were provided for every data run and configuration.

Pilots were administered the paired-comparison SWORD scale that enabled comparative ratings of mental workload. Mental workload was defined for the pilots as "the amount of cognitive resources available to perform a task and the difficulty of that task." The pair-comparison test was structured to compare the effects of peripheral cue (i.e., "visibility") and display type (i.e., VMC/HUD, IMC/HUD, VMC/XVS HUD FOV, IMC/XVS HUD FOV) flown by the EP. Note that this comparison does not include the effects of FOV. Only the HUD FOV configurations were considered. 


\subsubsection{NASA TLX}

Approach and Landing Operations: An ANOVA revealed that display $(\mathrm{F}(2,142)=9.64, \mathrm{p}<0.001)$ and peripheral cues $(\mathrm{F}(1,142)=9.02, \mathrm{p}=0.003)$ were highly significant for workload ratings. Post-hoc tests showed two unique subsets for workload ratings with the display concepts: 1) XVS with Full FOV (mean=30) having lower workload and 2) XVS with HUD FOV (mean=34) and HUD (mean=34) having higher workload. Pilots rated their workload significantly lower when they had peripheral cues (mean=30) on the approach compared to when they didn't have them (mean=33).

Surface Operations: An ANOVA revealed that display $(\mathrm{F}(2,139)=28.75, \mathrm{p}<0.001)$ and peripheral cues $(\mathrm{F}(1,139)=4.86$, $\mathrm{p}=0.029)$ were significant for workload ratings. Post-hoc tests showed three unique subsets for workload ratings: 1 ) the XVS with Full FOV concept (mean=24) having the lowest workload, 2) the XVS with HUD FOV concept (mean=28), and 3) the HUD concept (mean=33) with the highest workload during surface operations. Pilots rated their workload significantly lower when they had peripheral cues (mean=27) while taxiing compared to when they did not have them (mean=29).

Departure: An ANOVA revealed that the peripheral cues factor $(F(1,46)=6.01, p=0.018)$ was significant for pilot departure workload ratings, but display (mean $=29)$ was not significant $(\mathrm{p}>0.05)$ for this measure. Pilots rated their workload lower when they had peripheral cues $($ mean $=28)$ in the side windows during a departure compared to when they didn't have them (mean=30).

\subsubsection{SWORD}

The post-test paired-comparison SWORD data indicate that visibility/display type $(\mathrm{F}(3,92)=49.05, \mathrm{p}<0.001)$ was highly significant for the pilot ratings of mental workload. Post-hoc tests (SNK using $\alpha=0.05$ ) showed three unique subsets for the mental workload ratings with the 4 visibility/display combinations: 1) VMC/XVS HUD FOV (lowest workload); 2) IMC/XVS HUD FOV and VMC/HUD; and 3) IMC HUD (highest workload).

\subsubsection{Workload Discussion}

The subjective post-run ratings indicated the workload was significantly reduced when using the larger displays (i.e., greater FOV) and when peripheral cues were available OTW for approach and surface operations. In consideration of the mean rating differences however, the workload changes created by these effects are not operationally significant. In all cases, the pilot workload was very manageable when performing approach and landing using any of the evaluated concepts. This result is somewhat surprising for surface operations, since, in IMC conditions (200 RVR) and with the smaller FOV displays in particular, usable visual information is provided to the pilot only in very limited areas (the HUD and side windows). These surface operations results were likely influenced by the PI aiding the EP during right taxi turns.

During departures, no workload differences were found for display size (i.e., FOV) but there were workload improvements when peripheral cues were added. Even though statistically significant workload differences were found, operationally these differences were not substantial - all were ranked at a moderately low workload level on the TLX scale.

The subjective post-test ratings where display size was held fixed revealed that having color in the display was a larger influence in workload ratings than a collimated display. These results were supported by subjective commentary made during the post-test interviews.

\subsection{Situation Awareness}

Situation awareness was assessed after each experimental run, using the post-run SART, and post-test, using the SASWORD measures.

SART is a multi-dimensional rating technique using the constructs of: 1) demand on attentional resources; 2) supply of attentional resources; and, 3) understanding. From these components, the SART rating is "understanding" reduced by the difference of "demand" minus "supply" (i.e., SART $=\{$ (understanding $)-($ demand - supply $)\})$. SART ratings were provided for every data run and configuration.

Similar to the SWORD described above, the SA-SWORD is a paired-comparison technique that provides relative situation awareness ratings. For these comparisons, SA was defined as "the pilot's awareness and understanding of all factors that will contribute to the safe flying of their aircraft under normal and non-normal conditions." The paircomparison test was structured to compare the effects of peripheral cue (i.e., "visibility") and display type (i.e., 
VMC/HUD, IMC/HUD, VMC/XVS HUD FOV, IMC/XVS HUD FOV) flown by the EP. Note that this comparison does not include the effects of FOV. Only the HUD FOV configurations were considered. The SA-SWORD measure differs from the post-run SART measure construct as it is a pair-comparison test, it was administered post-test for all configurations, and the underlying definition and construct for the SA ratings are different.

\subsubsection{SART}

Approach and Landing Operations: An ANOVA revealed that display $(F(2,142)=10.94, p<0.001)$ and peripheral cues $(\mathrm{F}(1,142)=15.66, \mathrm{p}<0.001)$ were highly significant for pilot approach SART ratings. Post-hoc tests showed two unique subsets for SA ratings with the display concepts: 1) XVS with Full FOV (higher SA) and 2) XVS with HUD FOV and HUD (lowest SA). Pilots rated their SA significantly higher when they had peripheral cues on the approach compared to when they didn't have them.

Surface Operations: An ANOVA revealed that display $(\mathrm{F}(2,139)=47.46, \mathrm{p}<0.001)$ was highly significant for pilot taxi SART ratings, but peripheral cues was not significant $(\mathrm{p}>0.05)$ for this measure. Post-hoc tests showed three unique subsets for the SA ratings with the XVS with Full FOV concept providing the highest SA, followed by the XVS with HUD FOV concept, and then finally the HUD concept providing the least SA during surface operations.

Departure: An ANOVA revealed that display $(\mathrm{F}(2,46)=4.07, \mathrm{p}=0.024)$ was significant for pilot departure SART ratings, but peripheral cues was not significant $(\mathrm{p}>0.05)$ for this measure. Post hoc tests showed that the XVS with Full FOV concept provided higher SA than either the XVS with HUD FOV concept or HUD concept during a departure. There were no appreciable SA differences between these two smaller size display concepts.

\subsubsection{SA-SWORD}

The post-test SA-SWORD data indicate that visibility/display type $(F(3,92)=96.79, p<0.001)$ was highly significant for the pilot ratings of SA. Post-hoc tests (SNK using $\alpha=0.05$ ) showed four unique subsets for the SA ratings with the 4 visibility/display types: 1) VMC/XVS HUD FOV (highest SA); 2) IMC/XVS HUD FOV; 3) VMC/HUD; and 4) IMC HUD (lowest SA).

\subsubsection{Situation Awareness Discussion}

The subjective post-run ratings indicated that SA was significantly enhanced when using the larger displays compared to the smaller ones for approach and landing, surface, and departure operations. SA improvements due to having peripheral cues in the side windows was only realized during approach and landing operations. This result for surface operations is somewhat counter-intuitive given the importance of full-field of regard information for taxi path and traffic awareness on the airport but they, too, were probably influenced by the PI aiding the EP during surface operations.

Similar to the workload results, the subjective post-test ratings where display size was held fixed revealed that having color in the display was a larger influence on SA than a collimated display. These results were also supported by pilot comments during the post-test interviews.

\section{CONCLUSIONS}

An experiment was conducted to investigate the use of S/EV and XVS technologies as enabling technologies for future all-weather operations. The experimental objectives were to determine the feasibility of these advanced vision technologies to provide for all weather (visibility) landing capability without the need (or ability) for a visual approach segment and to determine the interaction of S/EV and XVS and peripheral vision cues for terminal area and surface operations.

Objective results indicate that elimination of the visual segment of the approach had no adverse affects on localizer tracking as it was excellent regardless of display size (large FOV or HUD FOV) or whether the pilot had peripheral cues or not. Elimination of the visual segment appears to have improved a pilot's glideslope tracking compared to previous experiments requiring a visual segment. This improvement can most likely be attributed to the fact that the pilots didn't have to simultaneously perform glideslope corrections and find required visual landing references in order to continue a landing. In addition, a larger display size appears to offer slight improvements in glideslope tracking over smaller size displays. Landings were completed safely regardless of display type. In fact, landings made with either color XVS display concept were within JAR AWO touchdown point criteria. 
Not surprising, subjective results indicate that a larger display size provides significantly more situation awareness during approach, surface, and departure operations than smaller size displays. Workload reductions during approach and landing operations were also found with the larger size displays compared to the smaller ones, but not during departure operations. Having peripheral cues in the side windows significantly reduced pilot workload during all operational phases tested, but these workload differences appear operationally inconsequential. Similar results for peripheral cues were found for SA. These results are somewhat surprising given the importance of full field-of-regard information for the surface operations task (e.g., awareness and maintenance of the taxi path; separation from other traffic; and ground hazards). Further investigation of these results is necessary.

When display size was held fixed, SA and workload improvements were seen with the color, uncollimated XVS display compared to the monochrome, collimated HUD display, according to paired-comparison tests. Subjective comments indicate that color was the biggest discriminator, not collimation, in these pilot ratings. This result is not surprising considering that a HUD is designed to operate in concert with the out the window view. Since the forward vision was obscured, the XVS provided this missing information.

The data showed little evidence of a display collimation effect (i.e., when comparing XVS-HUD vs. HUD). In this experiment, this effect was confounded by the display color differences, but there was little subjective commentary noting an influence of display collimation. Use of a motion-based simulator while conducting approach, landing and surface operations with these conformal head-up display concept using advanced vision technologies should be explored to evaluate the effects of motion on collimation, groundspeed, and ground rush cues.

\section{REFERENCES}

[1] Joint Planning and Development Office. (2004). Next generation air transportation system integrated plan. Washington, DC: Author.

[2] National Aeronautics and Space Administration. (n.d.). Aviation safety program/integrated intelligent flight deck technical plan summary. Retrieved October 3, 2007, from http://www.aeronautics.nasa.gov/nra_pdf/iifd_tech_plan_c1.pdf

[3] Bailey, R. E., Kramer, L. J., \& Prinzel, L. J., III. (2006). Crew and display concepts evaluation for synthetic/enhanced vision systems. Proceedings of SPIE, Enhanced and Synthetic Vision Conference 2006, 6226, 62260G.1-62260G.18.

[4] Bailey, R. E., Kramer, L. J., \& Prinzel, L., III. (2007). Fusion of synthetic and enhanced vision for all-weather commercial aviation operations (Tech. Rep. No. NATO RTO-HFM-141). In NATO Human Factors and Medicine Symposium on Human Factors and Medical Aspects of Day/Night All Weather Operations: Current Issues and Future Challenges (pp. 11-1-1118). Neuilly-sur-Seine, France: RTO.

[5] Kramer, L. J. and Norman, R. M. (2000). High-speed research surveillance symbology assessment experiment (Tech. Memo. No. NASA TM-2000-210107). Washington, DC: NASA.

[6] Kramer, L. J, Parrish, R. V, Williams, S. P., and Lavell, J. S. (1999). Effects of inboard horizontal field of view display limitations on pilot path control during total in-flight simulator (TIFS) flight test (Tech. Rep. No. NASA TP-1999-209542). Washington, DC: NASA.

[7] Kramer, L. J., Prinzel, L. J., III, Arthur, J. J., III, \& Bailey, R. E. (2005). Advanced pathway guidance evaluations on a synthetic vision head-up display (Tech. Rep. No. NASA TP-2005-213782).Washington, DC: NASA.

[8] Merrick, V.K. and Jeske, J.A. (1995). Flightpath synthesis and HUD scaling for V/STOL terminal area operations. (Tech. Memo. No. NASA TM-1995-110348). Washington, DC:NASA.

[9] AIAA (1993). Guide to Human Performance Measurement. Washington D.C.: American Institute of Aeronautics and Astronautics.

[10] Vidulich, M.A. and Hughes, E.R. (1991). Testing a subjective metric of situation awareness. In Proceedings of the Human Factors \& Ergonomics Society, $35^{\text {th }}$ Annual Meeting (pp. 1307-1311). Santa Monica, CA: Human Factors Society.

[11] Foyle, D.C and Hooey, B.L. (2003). Improving evaluation and system design through the use of off-nominal testing: A methodology for scenario development. In Proceedings of the 12th International Symposium on Aviation Psychology (pp. 397-402). Dayton, OH: Wright State University.

[12] Joint Aviation Authorities. (2007). Joint Aviation Requirements, All-Weather Operations (Amendment 4). Englewood, CO: Global Engineering Documents.

[13] Kramer, Lynda J.; Williams, Steven P.; \& Bailey, Randall E. (2008). Simulation evaluation of synthetic vision as an enabling technology for equivalent visual operations. In Proceedings of SPIE Enhanced and Synthetic Vision Conference 2008, 6957, 69570K-1-69570K-15. 